$5-5-2020$

\title{
Analysis of a Modification to the Sexual Experiences Survey to Assess Intimate Partner Sexual Violence
}

\author{
RaeAnn E. Anderson \\ University of North Dakota, raeann.anderson@UND.edu \\ Samantha C. Holmes \\ Nicole L. Johnson \\ Dawn M. Johnson
}

\section{How does access to this work benefit you? Let us know!}

Follow this and additional works at: https://commons.und.edu/psych-fac

Part of the Psychology Commons

\section{Recommended Citation}

RaeAnn E. Anderson, Samantha C. Holmes, Nicole L. Johnson, et al.. "Analysis of a Modification to the Sexual Experiences Survey to Assess Intimate Partner Sexual Violence" (2020). Psychology Faculty Publications. 31.

https://commons.und.edu/psych-fac/31

This Article is brought to you for free and open access by the Department of Psychology at UND Scholarly Commons. It has been accepted for inclusion in Psychology Faculty Publications by an authorized administrator of UND Scholarly Commons. For more information, please contact und.commons@library.und.edu. 
Analysis of a Modification to the Sexual Experiences Survey to Assess Intimate Partner Sexual Violence

RaeAnn E. Anderson ${ }^{1 *}$, Samantha C. Holmes ${ }^{2,3}$, Nicole L. Johnson ${ }^{4}$, Dawn M. Johnson ${ }^{2}$

1. University of North Dakota, Department of Psychology, 2000 Columbia Hall, Grand Forks, ND 58202

2. University of Akron, Department of Psychology, 290 E. Buchtel Ave, Akron OH 44325

3. Yale School of Medicine, Department of Psychology, 300 George Street, Suite 901, New Haven, CT 06511

4. Lehigh University, Department of Education and Human Services, Iacocca Hall, 111 Research Drive, Bethelem, PA 18015

*corresponding author, raeann.anderson@und.edu, phone: 701-777-6824, fax: 701-777-3454

First submitted: June $25^{\text {th }}, 2019$, Revision submitted: November, $26^{\text {th }}, 2019$, Second revision

submitted: February $20^{\text {th }}, 2020$, Accepted: May $5^{\text {th }}, 2020$

Body word count: 7905

Dr. Anderson's efforts were funded in part by was supported by a grant from the National Institute on Alcohol Abuse and Alcoholism 1K01AA026643-01A1. Dr. Holmes' efforts were supported by NIH grant T32 DA019426. The content is solely the responsibility of the authors and does not necessarily represent the official views of the funding agency 


\begin{abstract}
:
Greater accuracy is needed in the assessment of sexual victimization that occurs in intimate relationships. Existing assessment strategies in the literature often represent two distinct approaches - intimate partner violence specific strategies vs. sexual violence specific strategies. The current study compared multiple distinct strategies for assessing intimate partner sexual victimization (IPSV) and evaluated a modification that optimizes intimate partner and sexual violence specific strategies. Two samples of undergraduate women were recruited. Sample $1(N$ =236) completed the Severity of Violence Against Women Scales (SVAWS) and a modified version Sexual Experiences Survey-Short Form Victimization (SES-SFV) in which participants were cued to respond both for romantic partners and non-partners (referred to as the SES$\mathrm{RP} / \mathrm{NP})$. Sample $2(\mathrm{~N}=206)$ completed the SVAWS and was randomized to either the traditional SES-SFV or the SES-RP/NP. Across samples, the prevalence of IPSV varied based on the measure used $(\mathrm{SVAWS}=11.7 \%$; SES-SFV $=17.0 \%$; SES-RP/NP $=25.4 \%)$. The SESRP/NP identified significantly more IPSV than the SES-SFV, SVAWS, and prior studies. Both the SES-SFV and the SES-RP/NP were positively and significantly associated with the SVAWS. The results suggested that optimal measurement of IPSV would consider both intimate partner and sexual violence strategies.
\end{abstract}

Keywords: intimate partner violence, domestic violence, rape, sexual violence, sexual assault, measurement 
Analysis of a Modification to the Sexual Experiences Survey to Assess Intimate Partner Sexual Violence

Since the 1980s there have been many improvements in the science of measuring sexual victimization, or the experience of being harmed by sexual violence (Cook, Gidycz, Koss, Murphy, 2011). However, in spite of the revolution of behaviorally specific questions, there remains a need for greater accuracy and precision in violence research (Hamby, 2017a). Notwithstanding numerous calls for sexual violence research measurement, and even detailed research agendas (Cook et al., 2011; Koss et al., 2007), relatively little research has examined measurement issues in detail. For example, research focused on how differences in item wording and item structure may change response rates is highly informative but rare (for example, Abbey, Parkhill, \& Koss, 2005; Rueff \& Gross, 2017). Further, very few measurement-focused studies have examined intimate partner sexual violence (IPSV) specifically. The goal of this study was to examine different strategies for the measurement of IPSV by analyzing the number of cases of IPSV identified by two frequently used measures: the Sexual Experiences Survey-Short Form Victimization (SES-SFV; Koss et al., 2007) and the Severity of Violence Against Women Scales (SVAWS; Marshall, 1992). We also analyzed a modified SES-SFV designed to optimize the strengths of existing strategies. Although many available measures, including the SES-SFV and SVAWS, capture incidents beyond specific cases of IPSV, our paper will focus exclusively on IPSV.

Intimate partner violence (IPV) refers to any type of violence or abuse, be it emotional, physical, or sexual, perpetrated by an intimate partner; thus, IPV by definition includes a broad array of behaviors but is perpetrator specific. Intimate partners comprise a range of relationships such as spouses, boyfriends/girlfriends, dating partners, and ongoing sexual partners (Basile, Smith, Breiding, Black, \& Mahendra, 2014). Sexual violence is an umbrella term that refers to 
any sexual contact obtained without consent by any perpetrator (Basile et al., 2014); thus, this term is less broad concerning the type of behaviors but broader in terms of the relationship to the perpetrator. There are several forms of sexual violence, the most severe being rape; rape is sexual intercourse (oral, anal, vaginal) obtained via substance intoxication, threats of force, or physical force. Other forms of sexual violence include nonconsensual sexual contact (e.g., being groped) and verbal coercion, or sexual intercourse obtained by verbal pressure or criticism (Koss et al., 2007). Measuring IPSV, therefore, combines the challenges of these two important and related but historically distinct areas of violence research: intimate partner violence and sexual violence (Hamby, 2017b; Cook et al., 2011). Identifying cases of IPSV means differentiating cases of sexual violence perpetrated by non-partners such as acquaintances or strangers, as could be the goal in a consciousness-raising program where non-treatment seeking women may have difficulty recognizing coercion. Discriminating cases of IPSV from cases where severe physical IPV is present can also be difficult, for example, in the case of women in domestic violence shelters. Yet in both cases, not identifying IPSV may lead to a lack of needed services as IPSV has been associated with serious health consequences (Barker, Stewart, \& Vigod, 2019). Finally, we note that these definitions are behaviorally focused and somewhat de-contextualized given the Western worldview of much research; whereas, how these behaviors are recognized and interpreted by those who experience them has been very much grounded in a socio-cultural context (Torres, 2019).

\section{Significance}

People who experience IPSV may endure the most severe experiences of either sexual violence or IPV. Though hampered by methodological issues, research has suggested cases which include IPSV have the highest and most serious health needs, such as risk for homicide 
(Barker et al., 2019). IPSV was shown to be a common cause of teen and unintended pregnancies which are at greater risk for poorer health outcomes (Barber, Kusunoki, Gatny \& Melendez, 2017). It would appear that these cases have often not been recognized as sexual violence, broadly, or sexual violence within the context of IPV, since neither have been routinely screened for in clinical practice (Probst, Turchik, Zimak \& Huckins, 2011). Thus, survivors of IPSV may go unrecognized and untreated even in treatment seeking settings.

\section{Existing Approaches and Issues in Measuring Sexual Violence}

Generally speaking, in the area of sexual violence, both the original Sexual Experiences Survey (SES) and the subsequently revised short-form version, the SES-SFV, have been the dominant approach to measurement. The SES-SFV was designed to measure sexual victimization from any person and is not specific to IPSV; yet, by design, its all-perpetratorrelationships-included strategy should capture incidents of IPSV as well as other incidents. Both the SES and SES-SFV have been recommended measures of IPSV (Bagwell-Gray, Messing, Baldwin-White, 2015), perhaps because they are one of the most empirically supported measures available. Indeed, the SES/SES-SFV demonstrated better test-retest reliability than the Revised Conflicts Tactics Scales-sexual coercion (CTS2) subscale (Vega \& O’Leary, 2007; Johnson, Murphy, \& Gidycz, 2017), a measure of IPV that includes a sexual violence subscale (although the CTS2 measures multiple domains of IPV, in this paper, when the CTS2 is referenced we are referring to using it as a measure of IPSV). Further, as noted by Bagwell-Gray et al. (2015), the SES/SES-SFV captured the greatest range of sexual victimization of available measures, including IPV-based approaches. However, little research has examined the usefulness of the SES or SES-SFV as a measure of IPSV specifically. 
Research has shown that unintentional underreporting remains a problem for measuring sexual violence victimization and that this may be especially true when sexual violence occurs in intimate partnerships. Studies demonstrated that participants may have internalized rape myths such as that rape cannot occur within an intimate relationship and therefore inadvertently omit these experiences when completing questionnaires (Aguilar, Mahapatra, Busch-Armendariz, \& Dinitto, 2016; Ferro, Cermele, \& Saltzman, 2008), an idea Anderson et al. (2018) called the partner-exception hypothesis. They suggested the CTS2 instructions may act as an effective memory cue for experiences of IPSV. In other words, respondents may unwittingly complete the SES-SFV with a "partner exception" in mind and the cues to include partners on the CTS2 then increase responding by facilitating the recall of IPSV. Although it was shown that participants do not need to internally label sexually violent experiences as "rape" in order to report them on behaviorally-specific questionnaires such as the SES/SES-SFV (Fisher, Cullen, \& Daigle, 2005), it may be that to overcome these internalized myths, specific measurement strategies are needed, such as descriptive cues to trigger salient memories and consider romantic partners as initiators of perpetration behavior. This is consistent with research that demonstrated: more specific cues better facilitated recall (Fisher, 2009), people often endorsed rape myths such as believing that women in relationships cannot be raped by their partners (Ferro et al., 2008), and that men reported, in interviews, that they may be less likely to recognize their own perpetration behavior when it is against a partner (Aguilar et al., 2016).

Current recommendations for sexual violence measures reflect Fisher's (2009) and others' work regarding the specificity of cues and suggest that measures of sexual victimization should include three components at minimum: behaviorally specific descriptions of the sexual act, descriptions of the tactic used to coerce the sexual act, and assessment of consent (Cook et 
al., 2011). However, for measuring IPSV, it is notable that this minimum does not include relationship to the perpetrator and other contextual elements. Indeed, one review of the IPSV literature noted that definitions and operationalizations of IPSV were highly variable (Barker, Stewart, \& Vigod, 2019) and have hindered research on this topic. Researchers have suggested that the context of violence is important for understanding how people cope and recover after IPV. Thus, some recommend this contextual information should be included in standardized questionnaires. For example, relationship to the perpetrator has helped to underscore the complexity of IPV and provide information that may be helpful for understanding and facilitating resilience and recovery (Lindhorst \& Tajima, 2008). Yet, to our knowledge, no research has examined how cues related to the relationship of the perpetrator, which are embedded in questionnaires, may be related to differences in how participants interpret questionnaires and thus disclose their IPSV experiences

Taxonomic efforts such as those outlined by Cook and Parrott (2009), Bagwell-Gray et al., (2015) and elaborated on in Bagwell-Gray (2019), demonstrated the gaps in our measurement systems. In general, most available measures, including the SES/SES-SFV, do not capture passive forms of violence such as failing to inform a partner about sexually transmitted infections and other methods of sexual violence that are relatively non-invasive and non-forceful. Even considering questionnaires that are more well-researched, including the SES-SFV, most only capture active and forceful forms of violence.

Previous research comparing the SES or the SES-SFV to measures of IPSV highlighted important discrepancies that provide some clues to the importance of relationship cues, which are absent in the standard SES or SES-SFV items. One study of women in domestic violence shelters found that the CTS2 identified $73 \%$ of respondents as survivors of sexual violence while the 
SES-SFV identified 63\% of the sample (Moreau, Boucher, Hébert, \& Lemelin, 2014). Assuming the measures had equal sensitivity to detecting IPSV, one would expect the SES-SFV to detect more cases, as the SES-SFV should detect all cases that the CTS2 identifies as well as cases perpetrated by non-partners (i.e., family member, acquaintance. or stranger). Anderson et al. (2018) replicated this finding in a sample of college men - for both sexual victimization and sexual perpetration. Notably, Anderson et al. (2018) directly compared specific items from each measure that assessed the same constructs, and assessed order of administration effects for the questionnaires, to control for potential issues related to the SES-SFV assessing a broader range of tactics (with more items). They found no significant order effects for sexual victimization. Anderson et al. (2018) suggested the partner exception hypothesis as one of many possible explanations for the discrepancy between the SES (Cook, 2002), the SES-SFV (Moreau et al. 2014; Anderson et al. 2018), and the CTS2 in detecting IPSV. They also suggested that randomizing the order of items within questionnaires, the questionnaire response format, and other structural features of questionnaires may have contributed to these discrepancies, consistent with related literature on sexual violence perpetration measurement (Strang, Heiman, Hill, \& Peterson, 2013).

\section{Existing Approaches to Measuring IPV \& IPSV}

In contrast to measures of sexual violence, measures of IPV assess sexual violence as one of many possible ways an intimate partner may be harmed. One common tool is the CTS2 which has received much criticism (Ackerman, 2018; Hamby, 2017b; Lehrner \& Allen, 2014), particularly regarding potential over-reporting of physical assault by inadvertently eliciting falsepositives (i.e., results that indicate the presence of physical assault when in fact participants are referencing horseplay or joking, see Ackerman, 2018; Hamby, 2017b). It is unclear whether the 
design features that are associated with over-reporting of physical assault would apply to the sexual violence items. Indeed, inspection of the sexual violence items indicates there may be some misclassification for the item "[my partner] insisted on sex...(but did not use physical force)" which could reference verbal coercion or consensual but unwanted sex (i.e., sex that a person agreed to but did not desire sexually).

The SVAWS was developed in 1992 as a more gender sensitive alternative to the CTS2 that encompasses a wider range of potentially abusive behaviors (Marshall, 1992). Importantly, in the development study for the SVAWS participants rated the seriousness, abusiveness, threat level, and violence of each item (including sexual violence items) to ensure that items did not identify false positives that actually represented horseplay, jokes, or accidents. It was demonstrated that in the case of sexual violence, false positives could include consensual bondage, role-play, or sexual activities that were consensual but unwanted (Peterson \& Muehlenhard, 2007). Thus, the SVAWS was developed to assess sexual violence as one form in which women could be abused by male intimate partners, and the validity of items were specifically tested to eliminate items that might identify false-positives as has been a challenge with the CTS2.

However, as noted by Bagwell-Gray et al. (2015), in comparison to measures of IPV that include sexual violence as a domain (such as the CTS2 and the SVAWS), the SES/SES-SFV (a sexual violence specific strategy) assesses more types of IPSV and has more items with IPSV content, and is therefore recommended as the instrument of choice with modifications to better capture IPSV. Even so, utilizing the SES or SES-SFV with modifications to identify IPSV raises some concerns. Research has demonstrated that even very small changes in wording can create significantly different response rates. For example, different conceptualizations of consent, such 
as using the phrase "unwanted" instead of non-consensual have been interpreted differentially by participants (Hamby \& Koss, 2003). Altering the CTS2 to be nonspecific regarding partners, and instead identifying the perpetrator in follow-up questions, increased reporting of severe physical IPV for women compared with men (Hamby, 2014). Merely swapping the order of the clauses within SES items, such that the tactic of coercion is described before the sexual outcome, increased reporting of sexual victimization (Abbey et al., 2005). Thus, even small changes made in good faith may have consequences on prevalence rates and corresponding health correlates, demonstrating the importance of careful empirical exploration into the potential unintended effects of proposed changes.

In prior research using an IPV specific strategy (the CTS2) in a large college sample, Sabina and Straus (2008) found that $28 \%$ of the sample reported IPSV in the past year consistent with other estimates using the CTS2 (Anderson et al., 2018). In contrast, prior research using a sexual violence specific strategy (the SES with follow-up questions) in college women, found the prevalence of IPSV ranged from $3.0 \%$ (while on campus) to $6.5 \%$ (lifetime: Campbell et al. 2017; Littleton \& Henderson, 2009). Yet in their review, Bagwell-Gray et al. (2015) noted that the increased number of items on the SES/SES-SFV may increase reporting by capturing a greater number of types of incidents. The current literature is mixed regarding whether an IPVor sexual violence-specific strategy for assessing IPSV will result in higher prevalence rates. Thus, research that examines the specific effects of changes or compares different measurement strategies, particularly those that would change the construct being assessed (in this case, from sexual violence generally to IPSV specifically) is needed.

\section{The Current Study}


The current study compared two conceptually distinct strategies for assessing IPSV, an IPV specific strategy that considers sexual violence as one domain in which IPV may occur, and a sexual violence specific strategy, that considers romantic partners as one of many potential perpetrators of sexual violence. We also evaluated a third, distinct, strategy that follows the suggestion of Bagwell-Gray et al. (2015): We developed a partner cue modified SES-SFV, the SES-Romantic Partner/Non-Partner (SES-RP/NP), to specifically capture IPSV in a way that combines the strength of the research regarding the measurement of both IPV and sexual violence. Although the SES-RP/NP captures both intimate partner and non-partner sexual violence, this manuscript and subsequent analyses will focus exclusively on IPSV. Thus, we first compared the prevalence rates identified by the SES-SFV, the SVAWS, and the SES-RP/NP. In addition to documenting prevalence rates, we examined the degree of discrepancy in the number of cases identified by the different measures, used kappa to correct for agreement that occurs by chance, compared the strength of the correlation between the SES-RP/NP or the SES-SFV and the SVAWS, and assessed potential order effects.

Comparison of the SES-SFV and the SVAWS. We chose to compare the SES-SFV to the SVAWS due to SVAWS design features that are more similar to the structure of the SESSFV, and the decreased concern for false positive responses on the SVAWS compared with the CTS2. The questionnaires are also similar in that they use a hierarchical approach to the order of items (items increase in severity as the questionnaire progresses) and a frequency response scale, both questionnaire design features that have been shown to influence prevalence rates (Ramirez \& Straus, 2006; Hamby, Sugarman, \& Boney-McCoy, 2006). The SES-SFV contains many more items than the SVAWS, as can be seen by examining the example items in Table 1 and 3. Additionally, when a taxonomy of IPSV has been considered (Bagwell-Gray, 2019; Cook \& 
Parrott, 2009), the SVAWS seemed to mostly capture active or invasive (i.e., penetrative) and physically forceful acts, though one item does describe "demanded sex" but is ambiguous as to what this means behaviorally. In contrast, the SES-SFV captures invasive behaviors and both forceful and non-forceful behaviors by describing multiple ways that a participant may have been verbally pressured, bullied, emotionally manipulated, et cetera (see tactics a-b in Table 1). Because many of the SVAWS items are quite vague about specific tactics or sexual acts, we did not directly compare items between the questionnaires. Further, we did not expect complete correspondence between the questionnaires given these differences.

\section{Hypotheses (H)/Research Questions (RQ):}

RQ1 \& H1. We compared the number of cases of IPSV identified by the SES-SFV and the SVAWS in a sample of college women (Samples 2). We did not make a specific hypothesis regarding which measure would produce higher prevalence rates given that some research suggested there may be higher rates for the IPV-specific measures (Sabina and Straus, 2008) while other research suggested the same for the sexual violence-specific measure (Anderson et al., 2018; Bagwell et al., 2015). We hypothesized that agreement between the measures would be worse than that documented in prior literature (Moreau et al. 2014; Anderson et al., 2018) given the limited range of sexual content on the SVAWS. Specifically, we hypothesized that case agreement would be in the poor to moderate $(<60 \%$, kappa $<.4)$ range, which is less than that which has been demonstrated in prior research (>70\% Anderson et al., 2018; Moreau et al., 2014).

H2. We hypothesized that the SES-RP/NP would identify more cases of IPSV than the SVAWS in a non-experimental design (Samples $1 \& 2$; H2a). We hypothesized that the degree of agreement between the SES-RP/NP and the SVAWS would be moderate (approximately $60 \%$, 
kappa >.4, Sample $1 \& 2 ; \mathrm{H} 2 \mathrm{~b}$ ) given results from prior research (Anderson et al., 2018; Moreau et al., 2014).

H3. We hypothesized that the SES-RP/NP would identify more cases of IPSV than reported in previous, comparable studies that used the original SES or the SES-SFV (Samples 1 \& 2).

H4. We hypothesized that the SES-RP/NP would identify significantly more cases of IPSV than the standard SES-SFV in an experimental study where we randomly assigned participants to either the SES-RP/NP or the standard SES-SFV (Sample 2).

H5. Because there is no correction for measurement error or chance in percent agreement, we also computed correlations between the two measures as another assessment of the relationship between these measures. We hypothesized that there would be a significant positive correlation between the SES-RP/NP and the SES-SFV and the SVAWS (in the $r=.3$ to .4 range consistent with Davis et al., 2014). Further, we hypothesized that the strength of the correlations between the SES measures and the SVAWS would not significantly differ from each other (consistent with Anderson et al., 2018).

RQ2: Due to the lack of prior literature to guide hypotheses, we examined whether there are order effects dependent on if the SES-RP/NP was given before the SVAWS or vice-versa (Sample 2).

\section{Methods}

\section{Participants}

Sample 1. Sample 1 was initially recruited for a study on IPV, alcohol misuse, PTSD symptoms, and help-seeking behavior among undergraduate women but the inclusion of the SVAWS and the SES-RP/NP allowed for additional analyses related to measurement issues. The initial sample consisted of 322 undergraduate women; however, 75 participants did not meet the 
inclusion criteria (i.e., 18 years and older, identify as a woman, report being in an intimate relationship in the past year) and were consequently omitted from analyses. Of the remaining 247 participants, 11 did not complete any items on either the SES-RP/NP or the SVAWS.

The final sample comprised 236 undergraduate women, whose ages ranged from 18 to 31 years $(M=20.01, S D=1.99)$. The majority of the sample identified as White $(76.6 \%)$, followed by Black or African American (11.5\%), Multiracial (7.7\%), Asian or Pacific Islander (3.0\%), and Arab (1.3\%). Additionally, $7.6 \%$ of the sample identified as Hispanic or Latina. Most participants identified as exclusively heterosexual (79.7\%), and the remainder endorsed varying levels of same-sex attraction (20.3\%). With regard to socioeconomic status, $38.3 \%$ indicated their family has just enough money to buy what they need or less.

Sample 2. Sample 2 was recruited to test H3 and H4. Initially, Sample 2 comprised 314 participants; however, 95 did not meet the inclusion criteria (i.e., 18 years or older, identified as a woman, reported being in an intimate relationship in the past year) and were therefore removed. Of the remaining 219 participants, 10 had not completed any items on the SVAWS, SES-SFV, SES-RP/NP and one reported sexual violence on the SES-SFV but did not indicate their relationship with the perpetrator and, thus, were also omitted.

The final sample consisted of 208 participants who ranged from 18 to 26 years old $(M=$ 19.41, $S D=1.64)$. Most participants identified as White $(78.7 \%)$ followed by African American or Black (14.5\%), Multiracial (4.3\%), Asian or Pacific Islander (1.4\%), and Arab (1.0\%). In addition, $7.7 \%$ identified as Hispanic or Latina. The majority of participants identified as exclusively heterosexual (73.6\%), and the remainder (26.4\%) reported varying levels of samesex attraction. With regard to socioeconomic status, $27.4 \%$ indicated their family has just enough money to buy what they need or less. 


\section{Measures}

The SES-SFV (Koss et al., 2007). The SES-SFV for women consists of 10 total items. Seven of these are compound behaviorally specific items used to determine sexual victimization history: see Table 1 for an example item. The seven compound items begin with a sexual act as a stem and are followed by five sub-items (a-e) that describe the tactic used to coerce the sexual act, creating 35 items when crossing sexual act by tactics. Three of the sexual acts and all five of the tactic sub-items are listed in Table 1 . The remaining 4 sexual acts are described similarly and include attempted oral sex, attempted vaginal sex, anal sex, and attempted anal sex. Respondents completed the measure by responding to the number of times they have experienced each item $(0,1,2,3+)$ in the past twelve months. The items gradually increase in severity over the course of the instrument. The remaining three items are used to assess the context of sexual victimization (repeated victimization, gender of perpetrator, acknowledgment); data from these items are not presented in this study. There is good evidence of validity and adequate evidence of test-retest reliability for the SES-SFV in college women; SES-SFV scores were correlated to measures of psychological distress after trauma and scores were stable over a 1-2 week interval for $70 \%$ of participants (Johnson et al., 2017).

The SES-SFV was modified to create the SES-RP/NP in this study. For the SES-RP/NP, participants were queried about each SES-SFV item for two different relationship contexts: in an intimate relationship and not in an intimate relationship. Intimate relationships were defined as the following: "girlfriend, boyfriend, partner, wife, or husband" for at least one month. Nonintimate partners were defined as the following: "friend, acquaintance, stranger." These relationship contexts were presented side by side for each SES-SFV item: see Table 2 for an example. These two contexts can be combined to create an overall score or scores specific to 
each relationship type (SES-RP or SES-NP). We used prior literature to design this modification including Testa et al. (2007) and Ullman et al. (2006).

In Sample 1, participants completed the SES-RP/NP. In Sample 2 participants were randomly assigned to either the SES-RP/NP or the traditional SES-SFV. To identity IPSV via the SES-SFV we placed a follow-up question at the end: "What was your relationship to the person who did them to you?" Participants could choose one response from the following: intimate partner, friend, acquaintance, and stranger. Follow-up questions after the SES-SFV have been commonly used for identifying IPSV and other assault characteristics (Littleton \& Henderson, 2009; Jaffe, Steel, DiLillo, Messman-Moore, \& Gratz, 2017).

\section{The Severity of Violence Against Women Scales - Sexual Violence Subscale}

(SVAWS: Marshall, 1992). The SVAWS contains 46 items assessing a range of IPV victimization experiences: see Table 1 for the sexual violence subscale items. An intimate relationship was defined using the following examples: "girlfriend, boyfriend, partner, wife, or husband" for at least one month (i.e., in an identical manner to how it was defined on the SESs). The questionnaire begins with instructions intended to normalize potential IPV experiences: "During the past month, you and your partner(s) have probably experienced anger or conflict. Below is a list of behaviors you and your partner(s) may have done during the past month." Although participants responded to all 46 items, only items from the sexual violence subscale (6 items) were analyzed in this study; the six sexual violence items were administered at the end of the SVAWS, consistent with the way in which the measure was developed (Marshall, 1992). Participants rated each item on a 4-point scale from "never" to "many times," and we included an additional option, "not in the past month but in the past year" as a fifth response. All items begin with the phrase "your partner" and then describe a tactic and then a sexual act. The 
SVAWS has demonstrated good validity and clinical utility; SVAWS sexual violence scores have been related to a greater need for medical care, trauma symptoms, and risk for repeated victimization (McFarlane et al., 2005). We were unable to locate test-retest reliability data.

Scoring. For each measure we computed an overall dichotomous score (IPSV victimization: yes/no) to compare prevalence rates for the past year; any item scored 1 ('once'), or greater on the SES-RP/NP, SES-SFV, or SVAWS was considered an affirmative response. We used the same procedure to create continuous scores for the correlation analysis; any items that were endorsed $\geq 1$ was summed to create a continuous score. SVAWS continuous scores could thus range from 0-6 while SES-SFV and SES-RP/NP scores ranged from 0 to 35. Consistent with Davis et al. (2014), we computed multiple scores for H5 given that these measures are utilized in multiple ways in the literature. For any item that was scored "once" or greater, we added a value of one to the total frequency score. We did not calculate Cronbach's alpha for either measure, consistent with research that indicated that internal consistency is a good measure of reliability for latent constructs with normal distributions but inappropriate for measures of behavioral experiences (Diamantopoulos, Riefler, \& Roth, 2008; Koss et al., 2007; Hulme, 2007).

\section{Procedures}

Participants in both samples were recruited in two ways from a large public university in the Midwest. The first method was via the university's website where students enrolled in Introduction to Psychology courses can meet their course requirement of participating in research and students in other psychology courses can earn extra credit. Additionally, the current study was advertised through in-person classroom recruitment. Potential participants were told that the survey focused on intimate relationships and alcohol use among college women and were 
provided a link that brought them to a series of anonymous questionnaires on Qualtrics, an online software program. In Sample 1, data were collected as part of a larger study on IPV, trauma, and substance use; questionnaires regarding the following constructs were administered in a fixed order: demographics, unwanted pursuit and psychological maltreatment, SVAWS, SES-RP/NP, alcohol use and consequences, and, finally, posttraumatic stress disorder symptoms. In contrast, Sample 2 was specifically collected for the purposes of this study. For Sample 2, after completing demographics, questionnaires were administered in a randomized order and participants were randomly assigned to either the SES-RP/NP $(n=102)$ or the standard SESSFV $(n=106)$ as well as a questionnaire on alcohol use, and the SVAWS. All procedures were approved by the Institutional Review Board of the final author. At this time, data are available upon request from the final author. The dataset has not been posted publicly to respect the rights of research participants who did not consent to their data being posted on a public website.

\section{Power Analysis}

We computed a priori power analyses using G*Power (Faul, Erdfelder, Lang, \& Buchner, 2007) and consulted the results of Anderson et al., (2018) to estimate likely effect sizes for chi-square analyses. Power analyses suggested a total sample of $N=94$ to achieve Power $=$ .80 for effects of $w=.29$ (medium) size. We used the results of Davis et al., (2014) to estimate power needed for correlational analyses. Power analyses suggested a total sample of $N=133$ to achieve Power $=.80$ for weak effects $(r=.24)$. Following, the analyses presented in this paper are well-powered.

\section{Data Cleaning and Analytic Strategy}

Participants who had not completed any item on a version of the SES or on the SVAWS, or who endorsed sexual violence on the SES-SFV but did not indicate their relationship with the 
perpetrator, were omitted from analyses. If a participant completed at least one item on each measure, they were retained and any missing data were assumed to be the modal response (i.e., 0). This is consistent with Parent's (2013) demonstration that using available items is equivalent if not superior to multiple imputation and prior research suggesting the modal response rate for endorsing any individual IPSV item in healthy populations is zero (Sabina \& Straus, 2008; Littleton \& Henderson, 2009).

To address H1-4, cross-tabulations were computed comparing the number of participants identified as having an IPSV history on one measure to the number identified on the other. Concordance rates (i.e., percent agreement) were also determined by calculating the proportion of cases identified in the same way by both measures (i.e., participant was identified as having a sexual victimization history by both measures or by neither measure) to the overall number of cases (e.g., in Sample 1, $N=236$ ). Additionally, chi-squares were computed to address the null hypothesis that there would be no difference in prevalence rates between the two measures and kappa (к) to assess the rate of agreement while correcting for chance agreement. To calculate differences in prevalence rates from two different samples (H3), we utilized the "N-1" chi-square test as recommended by Campbell (2007) and Richardson (2011), which better accounts for low prevalence rates and different sample sizes. To assess for potential order effects (RQ2) that may have affected prevalence rates on the SESs and the SVAWS, we conducted a chi-square test to determine whether the possible patterns of concordance/discordance (both measures negative, SES positive/SVAWS negative, SES negative/SVAWS positive, both measure agree) varied as a function of whether the SES was administered first.

\section{Results}

\section{Descriptive Results}


When both Samples 1 and 2 were combined, prevalence of IPSV was $17.0 \%$ for the SES$\operatorname{SFV}(n=106), 25.4 \%$ for the SES-RP/NP $(n=338)$, and $11.7 \%$ for the SVAWS $(n=444)$. Frequencies of IPSV for each item on the SVAWS and the SESs can be found in Table 3. The following sections provide more detailed and direct comparisons among measures. Note, although we report results of the SES-RP/NP using the full name of the measure, given the focus of the paper, these scores represent only the cases of IPSV identified by the measure. In other words, the scores below only include cases perpetrated by intimate partners.

Research Question 1/Hypothesis 1: We compared the proportion of cases of IPSV identified by the SVAWS with the proportion of cases identified by the standard SES-SFV, using only Sample 2. We limited this to a direct comparison of participants who were administered both measures. The SVAWS $(n=22 / 106,20.8 \%)$ identified significantly more cases of IPSV in the past year than the SES-SFV $(n=18 / 106,17.0 \%), \chi^{2}(1)=4.34, p=.037$, Cramer's $V=.20$. Percent agreement was 75.5\% (80/106) for past year sexual victimization. Additionally, к was .20 , indicative of little to no agreement (McHugh, 2012).

Hypothesis 2a: Using data from Samples 1 and 2, the SES-RP/NP $(n=86 / 338,25.4 \%)$ identified significantly more cases of sexual victimization in the past year than the SVAWS ( $n=$ $30 / 338,8.9 \%), \chi^{2}(1)=34.45, p<.001$, Cramer's $V=.32$.

Hypothesis 2b: Percent agreement between the SES-RP/NP and SVAWS, across the two samples, was $78.1 \%$ (264/338) for past year sexual victimization. The rate of discordance was statistically significant, $\chi(1)=34.45, p<.001$, demonstrating that there is a relationship between sexual victimization and measure utilized (note this is the same chi-square analysis as $\mathrm{H} 2 \mathrm{a}$ but interpreted in relation to a different question). Additionally, к was .27, indicative of minimal agreement (McHugh, 2012). 
Hypothesis 3: We compared the number of cases of IPSV identified by the SES-RP/NP in Sample $1(22.9 \%, n=54 / 236)$ and Sample $2(31.4 \%, n=32 / 102)$ with the rate of IPSV reported in comparable literature using the standard SES-SFV approach, specifically, 6.5\% $(n=$ 114/1744: Littleton \& Henderson, 2009), and 3.0\% ( $n=35 / 1149$ : Campbell et al., 2017). Both prevalence rates detected by the SES-RP/NP were statistically larger than those documented by Littleton and Henderson (2009), $\chi^{2}(1) \geq 72.20, p<.001$ and Campbell et al. (2017), $\chi^{2}(1) \geq$ $129.51, p<.001$, Cramer's V $=.198$ using the SES-SFV with follow-up questions about the relationships to the perpetrator.

As a point of comparison, we also compared rates of IPSV as measured by the traditional SES-SFV in Sample $2(n=18 / 106,17.0 \%)$ to comparable literature to assess whether our sample perhaps differed from prior work. We found that the prevalence detected by the SES-SFV in the current study was statistically larger than those documented by Littleton and Henderson (2009), $\chi^{2}(1) \geq 16.69, p<.001$, and by Campbell et al. (2017), $\chi^{2}(1)=47.43, p<.001$ suggesting IPSV was higher in these samples regardless of measurement strategy.

Hypothesis 4: In Sample 2, there was a significant difference in the rate of IPSV identified based on the version of the SES to which participants were randomly assigned $\chi^{2}(1)=$ $5.87, p=.015$, Cramer's V $=.167$. Specifically, the SES-RP/NP $(31.4 \% ; 32 / 102)$ identified a significantly greater proportion of IPSV than the standard SES-SFV $(17.0 \% ; 18 / 106)$ with follow-up questions about the relationships to the perpetrator.

Hypothesis 5: In Sample 1, the SES-RP/NP was significantly correlated with the SVAWS both when scored dichotomously, $r(236)=.34, p<.001$, and when scored continuously, $r(236)=.56, p<.001$. In Sample 2 , the SES-RP/NP was similarly significantly correlated with the SVAWS [dichotomously: $r(102)=.27, p=.005$; continuously: $r(102)=.44$, 
$p<.001$ ], as was the SES-SFV with the SVAWS [dichotomously: $r(106)=.20, p=.038$;

continuously, $r(106)=.53, p<.001]$. We computed $z$ tests to determine whether the SES-RP/NP or the SES-SFV was more strongly correlated to the SVAWS in Sample 2. The strength of the correlations did not significantly differ [dichotomously: $z=.52, p=.598$; continuously: $z=-.84$, $p=.402]$.

Research Question 2: In Sample 2, the order in which the measures were administered was not related to the pattern of discordance between the SVAWS and SES-RP/NP, $\chi^{2}(3)=1.69$, $p=.639$, Cramer's $V=.13$, or the pattern of discordance between the SVAWS and SES-SFV, $\chi^{2}(3)=4.43, p=.219$, Cramer's $V=.20$.

\section{Discussion}

Despite calls for greater attention to the measurement of violence in research, very little research has examined IPSV measurement issues in detail. Indeed, current literature has noted that the SES/SES-SFV could be used with modification to assess IPSV because of the comprehensiveness of the SES/SES-SFV in assessing a continuum of sexual victimization (Bagwell-Gray et al., 2015). In contrast, other research documented that the SES/SES-SFV may underestimate IPSV, perhaps because of the lack of cues that regard partners as perpetrators or the design features of the questionnaires (Anderson et al., 2018) and incomplete assessment of the IPSV taxonomy (Bagwell-Gray, 2019). Thus, individual investigators are left to muddle through, lacking specific data from which to make decisions. This study sought to improve the measurement of IPSV by comparing three distinct strategies for measuring IPSV.

Following, in RQ1, we compared the rates of IPSV detected by the SVAWS, SES-SFV, and SES-RP/NP across the two current studies with the SES-SFV representing a sexual violence specific strategy, the SVAWS representing an IPV specific strategy, and the SES-RP/NP 
representing a third approach that optimizes the two former strategies. Interestingly, we found that the SES-RP/NP (25.4\%) and SVAWS (20.8\%) were superior to the SES-SFV (17.0\%) in detecting IPSV and there were no order effects (RQ2). While this is consistent with prior literature, in that when cued to regard romantic partners (as in the SES-RP/NP and the SVAWS), reported IPSV rates increased, it is remarkable given that the behavioral content of the SESRP/NP and the SES-SFV are identical. This finding also suggests that previous estimates of the scope of IPSV are likely inaccurate and further work is needed to establish how common IPSV is.

It is also noteworthy that all three prevalence rates were much higher than those reported in prior literature using any version of the SESs (consistent with H3) or any behaviorally specific measure with follow-up items, thus, our samples may have been unique. However, these prevalence rates were much closer to prevalence rates reported using the CTS2 in a large national sample of college students (28\%; Sabina \& Straus, 2008). This suggests the optimal measurement strategy for detecting IPSV may be a balance of the two distinct approaches (IPV specific vs. sexual violence specific) assessed here and as operationalized in this study by the SES-RP/NP.

Indeed, the modifications to the SES-SFV items made to specifically cue participants to consider romantic partners as perpetrators (the SES-RP/NP in this study), rather than using a follow-up question as in the standard SES-SFV, increased reporting of IPSV. This comparison was significant both compared with prior literature (SES-RP/NP: 22.9-31.4\% vs. SES-SFV: 3.0$6.5 \%$ ) and with the standard versions of the SES-SFV in Sample 2 (SES-RP/NP: 31.4\% vs. SESSFV: $17.0 \%$ ), consistent with H4. It appears that without a specific cue to regard romantic 
partners as perpetrators (e.g., the partner-exception hypothesis), participants may unintentionally under-disclose IPSV.

Correlations between the SES-RP/NP and the SVAWS were of weak to moderate strength and stronger when scored continuously. This provides some preliminary evidence of the validity of the SES-RP/NP. However, correlations between the SES-RP/NP and the SVAWS were not statistically different than the correlations between the SES-SFV and the SVAWS, although this may have been limited by the small sample size in Sample 2.

Further, the low concordance and kappa values between the SVAWS and the SESs used in this study are worth noting. Although percent agreement appeared healthy and better than hypothesized in $\mathrm{H} 1$ and $\mathrm{H} 2$ (75.5-78.1\%), and was similar (Moreau et al., 2012) or higher (Anderson et al., 2018) than prior literature, kappa values fell into a range that suggests the SVAWS and the SESs are measuring two different constructs. Indeed, following the BagwellGray (2019) taxonomy, the SVAWS assesses intimate partner sexual assault while the SESRP/NP assesses intimate partner sexual assault as well as sexual coercion and some aspects of physically forced sexual activity. This further underscores the variety in current approaches and lack of standardization in assessment tools to understand the scope of IPSV (Barker et al., 2019).

Notably, agreement was better comparing the two measures that included partner cues, the SVAWS to the SES-RP/NP, rather than the SES-SFV, again indicating support for the development of specific strategies to assess IPSV. It is also possible that low concordance was related to a mismatch in the rate of false-positives on the SVAWS and the SESs. Recent research suggested that up to $13 \%$ of SES-SFV responses may represent false positives, including incidents that may be consensual (Littleton, Layh, Rudolph, \& Haney, 2018), while the SVAWS was designed to minimize false positives (though we are unaware of further empirical tests of 
this premise). Finally, it is possible that discordance was related to other design features of the SVAWS and the SES-SFV and SES-RP/NP such as the differences in the conceptualization of consent in the two measures.

\section{Clinical and Research Implications}

The current study highlighted several clinical implications for working with survivors of IPV. Assessment and identification of abuse have been shown to be key factors in treating survivors of IPV, yet despite the high prevalence of sexual violence, they have not been routinely assessed in clinical practice (Probst, Turchik, Zimak, \& Huckins, 2011). At minimum, the current study highlighted the importance of prompting individuals to consider sexual violence within intimate relationships. This is of particular importance as research has identified significant relationships between IPSV and negative mental and physical health outcomes, as well as chronicity and severity of abuse (e.g., Littleton \& Henderson., 2009) suggesting survivors of IPSV may have especially high clinical needs (Barker et al., 2019). Furthermore, the SESRP/NP may be a useful resource for clients to assist with insight and consciousness raising, both of which may be precursors for therapeutic change, as cultural myths surrounding rape (e.g., a romantic partner cannot rape another partner) may impede personal labeling of IPSV. Finally, the SES-RP/NP may be a helpful educational tool for clients and therapists alike to increase specificity and understanding of IPSV.

In addition to clinical implications, the current results also suggest previous research may dramatically underestimate the prevalence of IPSV, particularly in relatively healthy samples. Further research on the measurement of IPSV is greatly needed. While reviewing literature for this article, we found very little research investigating optimal measurement strategies for IPSV either in terms of comparing existing measurement strategies or even ascertaining the test-retest 
reliability of common measures, much less experimentally testing various specific issues or hypotheses. We hope this paper, and the many others cited herein, inspires further research on IPSV measurement and related issues. For example, Bagwell-Gray (2019) suggested new items that capture a broader set of IPSV experiences and Straus and Douglas (2017) presented additional sexual violence items for the CTS2.

It appears that increasing specification of the relationship to the perpetrator, while simultaneously presenting information on the sexual acts and tactics, is important for identifying IPSV. While this is consistent with Fisher's (2009) work, it is somewhat at odds with work that has suggested that some of the SES-SFV items, particularly those assessing verbal coercion, are too long or detailed, resulting in confusion (Strang \& Peterson, 2017). Indeed, the verbal coercion item is nearly twice as long as the alcohol item (see Table 2). The length of this item may induce fatigue and thus a short-cutting or nay-saying response set. Thus, further research is needed to assess the balance between specificity in items and participant fatigue and confusion.

It is also possible that these findings, suggesting participants need specific cues for experiences that are inconsistent with rape myths, extend to other issues in sexual victimization measurement. For example, it may be that in incidents in which people experience tonic immobility during an assault (e.g., reflexive, involuntary paralysis) or explicitly choose not to fight back physically, they may also unintentionally underreport their experiences on sexual victimization questionnaires because these experiences are discrepant with rape scripts/stereotypes. Similarly, men who are raped by women and people who are coerced via verbal strategies may struggle to recognize these experiences as rape or sexual assault and thus, underreport their experiences. An important area for future research is further examining IPSV in a variety of relationship types, genders, and sexual identities. 
Future research should attempt to replicate our findings given that our results may have been influenced by the higher than usual rate of IPSV in our sample and other potential measurement differences between the SESs and the SVAWS. For example, the SESs mention consent very specifically using the phrase "without my consent" while the SVAWS uses a variety of phrases such as "against your will" and "whether you wanted it or not" which have been demonstrated to have differential meanings (Hamby \& Koss, 2003). It is important to know how modifications such as those made to the SES-RP/NP change the psychometrics properties of the SES-SFV on which it was based, particularly test-retest reliability. Finally, it is important to consider measurement within a taxonomic system and we encourage future research to explore how measures like the SES-RP/NP relate to taxonomies such as those suggested by BagwellGray (2019) and Cook and Parrot (2009).

\section{Limitations}

First, both samples exclusively comprised undergraduate women. Although college women were selected for their high rates of sexual victimization (Anderson et al., 2018; Holmes \& Johnson, 2017), this method limits the generalizability of our findings. In some ways, our findings of higher reported rates of IPSV were even more remarkable, given that prevalence rates have been shown to be typically smaller for brief periods of time (Johnson et al., 2017). However, Littleton and Henderson's (2009) study also exclusively examined rape whereas the current study assessed sexual victimization more broadly defined (i.e., including any unwanted sexual contact), which may contribute to the higher rates of IPSV detected in the current study. The way the study was advertised (e.g., mention of alcohol) may have also led to some selfselection of IPV survivors.

Another limitation is the way in which IPSV was measured by the traditional SES-SFV in 
Sample 2. As previously stated, the behavior items were followed by the question, "What was your relationship to the person who did them to you?" to which participants were able to select “intimate partner," “friend," "acquaintance” or "stranger." However, participants were only able to select one response; this approach appears somewhat typical in the literature and selective research has only recently illustrated how common revictimization and polyvictimization is to suggest this approach is overly limiting (Hamby \& Grych, 2013). Consequently, if participants had experienced sexual victimization by both a partner and someone of another relationship, they were only able to communicate one or the other, which may have resulted in an artificial restriction of IPSV rates detected by the SES-SFV. In spite of this limitation, the rates of IPSV documented in this study were still higher than those reported in prior literature.

In addition, 21 participants responded to this follow-up question regarding relationship to the perpetrator without endorsing any of the previous behavioral items. It is unclear whether these participants did, in fact, experience sexual victimization that was not captured by the behavioral items of the SES-SFV or if they were merely carelessly responding. Consequently, future research should replicate the results of the current study in larger samples that are more diverse regarding race, sexual orientation, and age, and allow participants to select multiple options when assessing perpetrator relationship on the SES-SFV.

\section{Conclusions}

Using the strengths of both IPV and sexual violence research is critical for accurately capturing cases of IPSV. The current study provided support for strategies that cue participants to consider romantic partners as potential perpetrators, as using the SES-RP/NP significantly increased rates of detected IPSV. Notably, we made very small changes to the actual text of the SES-RP/NP and the behavioral content remained identical - all while still significantly 
increasing prevalence rates. Consequently, it is possible that previously established rates of sexual victimization may have been underestimated if measures did not cue for romantic partners. Our findings also suggest that the scope of IPSV is likely dramatically under-estimated and more common than preciously suspected. The current study's modification of the SES-SFV (i.e., SES-RP/NP) may provide a useful tool for both clinicians and researchers who assess sexual victimization. Future research should therefore establish the psychometric properties of this version of the SES. 


\section{References}

Abbey, A., Parkhill, M. R., \& Koss, M. P. (2005). The effects of frame of reference on responses to questions about sexual assault victimization and perpetration. Psychology of Women Quarterly, 29(4), 364-373. http://doi.org/10.1111/j.1471-6402.2005.00236.x

Ackerman, J. (2018). Assessing Conflict Tactics Scale validity by examining intimate partner violence overreporting. Psychology of Violence, 8(2), 207-217. https://doi.org/10.1037/vio0000112

Anderson, R. E., Cahill, S. P., \& Delahanty, D. L. (2018). Discordance between the Sexual Experiences Surveys-Short Forms and the Revised Conflict Tactics Scales in college men. Psychology of Violence. https://doi.org/10.1037/vio0000199

Aguilar, J., Mahapatra, N., Busch-Armendariz, N., \& Dinitto, D. M. (2016). Exploring the science of conducting surveys for studying men's sexual assault perpetration behaviors. Journal of Aggression, Maltreatment \& Trauma, 25(1), 88-109. http://doi.org/10.1080/10926771.2015.1107171

Bagwell-Gray, M. E., Messing, J. T., \& Baldwin-White, A. (2015). Intimate partner sexual violence: A review of terms, definitions, and prevalence. Trauma, Violence, \& Abuse, 16(3), 316-335. http://doi.org/10.1177/1524838014557290

Bagwell-Gray, M. E. (2019). Women's experiences of sexual violence in intimate relationships: Applying a new taxonomy. Journal of Interpersonal Violence, 088626051982766. https://doi.org/10.1177/0886260519827667

Barker, L. C., Stewart, D. E., \& Vigod, S. N. (2019). Intimate partner sexual violence: An often overlooked problem. Journal of Women's Health, 28(3), 363-374. https://doi.org/10.1089/jwh.2017.6811 
Basile, K. C., Smith, S., Breiding, M. J., Black, M. C., \& Mahendra, R. (2014). Sexual Violence Surveillance: Uniform Definitions and Recommended Data Elements, Version 2.0. Retrieved from https://www.cdc.gov/violenceprevention/pdf/sv_surveillance_definitionsl2009-a.pdf

Campbell, I. (2007). Chi-squared and Fisher-Irwin tests of two-by-two tables with small sample recommendations. Statistics in Medicine, 26(19), 3661-3675. https://doi.org/10.1002/sim.2832

Campbell, J. C., Sabri, B., Budhathoki, C., Kaufman, M. R., Alhusen, J., \& Decker, M. R. (2017). Unwanted sexual acts among university students: Correlates of victimization and perpetration. Journal of Interpersonal Violence, 088626051773422. https://doi.org/10.1177/0886260517734221

Cook, S. L. (2002). Self-reports of sexual, physical, and nonphysical abuse perpetration. Violence Against Women, 8(5), 541-565. https://doi.org/10.1177/107780102400388425

Cook, S. L., Gidycz, C. A., Koss, M. P., \& Murphy, M. (2011). Emerging issues in the measurement of rape victimization. Violence Against Women, 17(2), 2012-18. http://doi.org/10.1177/1077801210397741

Davis, K. C., Gilmore, A. K., Stappenbeck, C. A., Balsan, M. J., George, W. H., \& Norris, J. (2014). How to score the Sexual Experiences Survey? A comparison of nine methods. Psychology of Violence, 4(4), 445-461. https://doi.org/10.1037/a0037494

Diamantopoulos, A., Riefler, P., \& Roth, K. P. (2008). Advancing formative measurement models. Journal of Business Research, 61(12), 1203-1218. http://doi.org/10.1016/j.jbusres.2008.01.009 
Dietz, T. L., \& Jasinski, J. L. (2007). The effect of item order on partner violence reporting: An examination of four versions of the revised Conflict Tactics Scales. Social Science Research, 36(1), 353-373.

Faul, F., Erdfelder, E., Lang, A. G., \& Buchner, A. (2007). G* Power 3: A flexible statistical power analysis program for the social, behavioral, and biomedical sciences. Behavior Research Methods, 39(2), 175-191.

Ferro, C., Cermele, J., \& Saltzman, A. (2008). Current perceptions of marital rape. Journal of Interpersonal Violence, 23(6), 764-779. http://doi.org/10.1177/0886260507313947

Fisher, B. S. (2009). The effects of survey question wording on rape estimates: Evidence from a quasi-experimental design. Violence Against Women, 15(2), 133147. http://doi.org/10.1177/1077801208329391

Fisher, B. S., Cullen, F. T., \& Daigle, L. E. (2005). The discovery of acquaintance rape: The salience of methodological innovation and rigor. Journal of Interpersonal Violence, 20(4), 493-500. https://doi.org/10.1177/0886260504267761

Hamby, S. (2014). Self-report measures that do not produce gender parity in intimate partner violence: A multi-study investigation. Psychology of Violence, 6(2), 323-335. https://doi.org/10.1037/a0038207

Hamby, S. (2017a). On defining violence, and why it matters. Psychology of Violence, 7(2), 167-180. http://doi.org/10.1037/vio0000117

Hamby, S. (2017b). A scientific answer to a scientific question: The gender debate on intimate partner violence. Trauma, Violence, \& Abuse, 18(2), 145-154. http://doi.org/10.1177/1524838015596963 
Hamby, S., \& Grych, J. (2013). Tracing the threads of the web: The epidemiology of interconnections among forms of violence and victimization. In The Web of Violence: Exploring Connections Among Different Forms of Interpersonal Violence and Abuse (pp. 8-27). New York, NY, US: Springer Netherlands. https://doi.org/10.1007/978-94-0075596-3_2

Hamby, S. L., \& Koss, M. P. (2003). Shades of gray: A qualitative study of terms used in the measurement of sexual victimization. Psychology of Women Quarterly, 27(3), 243-255. http://doi.org/10.1111/1471-6402.00104

Hamby, S., Sugarman, D. B., \& Boney-McCoy, S. (2006). Does questionnaire format impact reported partner violence rates?: An experimental study. Violence and Victims, 21(4), 507.

Holmes, S. C., \& Johnson, D. M. (2017). Applying objectification theory to the relationship between sexual victimization and disordered eating. The Counseling Psychologist, 45, 1091-1114. doi:10.1177/0011000017745977

Koss, M. P., Abbey, A., Campbell, R., Cook, S., Norris, J., Testa, M., ... White, J. (2007). Revising the SES: A Collaborative process to improve assessment of sexual aggression and victimization. Psychology of Women Quarterly, 31(4), 357-370. http://doi.org/10.1111/j.1471-6402.2007.00385.x

Jaffe, A. E., Steel, A. L., DiLillo, D., Messman-Moore, T. L., \& Gratz, K. L. (2017). Characterizing sexual violence in intimate relationships: An examination of blame attributions and rape acknowledgment. Journal of Interpersonal Violence, 088626051772697. https://doi.org/10.1177/0886260517726972 
Johnson, S. M., Murphy, M. J., \& Gidycz, C. A. (2017). Reliability and validity of the Sexual Experiences Survey-Short Forms Victimization and Perpetration. Violence and Victims, 32(1), 78-92. http://doi.org/10.1891/0886-6708.VV-D-15-00110

Lehrner, A., \& Allen, N. E. (2014). Construct validity of the Conflict Tactics Scales: A mixedmethod investigation of women's intimate partner violence. Psychology of Violence. Educational Publishing Foundation. https://doi.org/10.1037/a0037404

Lindhorst, T., \& Tajima, E. (2008). Reconceptualizing and operationalizing context in survey research on intimate partner violence. Journal of Interpersonal Violence, 23(3), 362-388. http://doi.org/10.1177/0886260507312293

Littleton, H., \& Henderson, C. E. (2009). If she is not a victim, does that mean she was not traumatized?: Evaluation of predictors of PTSD symptomatology among college rape victims. Violence Against Women, 15(2), 148-167. https://doi.org/10.1177/1077801208329386

Littleton, H., Layh, M., Rudolph, K., \& Haney, L. (2018). Evaluation of the Sexual Experiences Survey—Revised as a screening measure for sexual assault victimization among college students. Psychology of Violence. https://doi.org/10.1037/vio0000191

Marshall, L. L. (1992). Development of the Severity of Violence Against Women Scales. Journal of Family Violence, 7(2), 103-121. http://doi.org/10.1007/BF00978700

McFarlane, J., Malecha, A., Watson, K., Gist, J., Batten, E., Hall, I., \& Smith, S. (2005). Intimate partner sexual assault against women: Frequency, health consequences, and treatment outcomes. Obstetrics \& Gynecology, 105(1), 99-108. http://doi.org/10.1097/01.AOG.0000146641.98665.b6 
McHugh, M. L. (2012). Interrater reliability: the kappa statistic. Biochemia Medica, 22(3), 276282. Retrieved from http://www.ncbi.nlm.nih.gov/pmc/articles/PMC3900052/

Moreau, C., Boucher, S., Hébert, M., \& Lemelin, J. (2014). Capturing sexual violence experiences among battered women using the Revised Sexual Experiences Survey and the Revised Conflict Tactics Scales. Archives of Sexual Behavior, 44(1), 223-231. http://doi.org/10.1007/s10508-014-0345-8

Peterson, Z. D., \& Muehlenhard, C. L. (2007). Conceptualizing the "wantedness" of women's consensual and nonconsensual sexual experiences: Implications for how women label their experiences with rape. Journal of Sex Research, 44(1), 72-88. https://doi.org/10.1080/00224490709336794

Probst, D. R., Turchik, J. A., Zimak, E. H., \& Huckins, J. L. (2011). Assessment of sexual assault in clinical practice: Available screening tools for use with different adult populations. Journal of Aggression, Maltreatment \& Trauma, 20(2), 199-226. https://doi.org/10.1080/10926771.2011.546754

Ramirez, I. L., \& Straus, M. A. (2006). The effect of question order on disclosure of intimate partner violence: An experimental test using the Conflict Tactics Scales. Journal of Family Violence, 21(1), 1-9. https://doi.org/10.1007/s10896-005-9000-4

Richardson, J. T. E. (2011). The analysis of $2 \times 2$ contingency tables-yet again. Statistics in Medicine, 30(8), 890-890. https://doi.org/10.1002/sim.4116

Rueff, W. T., \& Gross, A. M. (2017). Assessing sexual coercion: Survey wording differences and the victimization-perpetration discrepancy. Journal of Family Violence, 32(3), 325-331. https://doi.org/10.1007/s10896-016-9859-2 
Ryan, K. M. (2013). Issues of reliability in measuring intimate partner violence during courtship. Sex Roles, 69(3-4), 131-148. https://doi.org/10.1007/s11199-012-0233-4

Sabina, C., \& Straus, M. A. (2008). Polyvictimization by dating partners and mental health among US college students. Violence and Victims, 23(6), 667-682.

Strang, E., Peterson, Z. D., Hill, Y. N., \& Heiman, J. R. (2013). Discrepant responding across self-report measures of men's coercive and aggressive sexual strategies. Journal of Sex Research, 50(5), 458-469. https://doi.org/10.1080/00224499.2011.646393

Strang, E., \& Peterson, Z. D. (2017). Unintentional misreporting on self-report measures of sexually aggressive behavior: An interview study. The Journal of Sex Research, 1-13. http://doi.org/10.1080/00224499.2017.1304519

Straus, M. A., \& Douglas, E. M. (2017). Eight new developments, uses, and clarifications of the Conflict Tactics Scales. Journal of Family Issues, 38(14), 1953-1973. https://doi.org/10.1177/0192513X17729720

Testa, M., VanZile-Tamsen, C., \& Livingston, J. A. (2007). Prospective prediction of women's sexual victimization by intimate and nonintimate male perpetrators. Journal of Consulting and Clinical Psychology, 75(1), 52-60. https://doi.org/10.1037/0022-006X.75.1.52

Ullman, S. E., Filipas, H. H., Townsend, S. M., \& Starzynski, L. L. (2006). The role of victimoffender relationship in women's sexual assault experiences. Journal of Interpersonal Violence, 21(6), 798-819.

Vega, E. M., \& O'Leary, K. D. (2007). Test-retest reliability of the revised Conflict Tactics Scales (CTS2). Journal of Family Violence. http://doi.org/10.1007/s10896-007-9118-7 
Table 1.

Comparison of SES-SFV and SVAWS items

SVAWS sexual violence subscale

SES-SFV items*

41. Your partner demanded sex whether you wanted it or not

42. Your partner made you have oral sex against your will

43. Your partner made you have sexual intercourse against your will

44. Your partner physically forced you to have sex

45. Your partner made you have anal sex against your will

46. Your partner used an object on you in a sexual way against your will.
3: If you are a male, check box and skip to item 4 . A man put his penis into my vagina, or someone inserted fingers or objects without my consent by: a: telling lies, threatening to end the relationship, threatening to spread rumors about me, making promises I knew were untrue, or continually verbally pressuring me after I said I didn't want to

b: showing displeasure, criticizing my sexuality or attractiveness, getting angry but not using physical force, after I said I didn't want to c: Taking advantage of me when I was too drunk or out of it to stop what was happening. $\mathrm{d}$ : Threatening to physically harm me or someone close to me.

e: Using force, for example holding me down with their body weight, pinning my arms, or having a weapon.

Note. SVAWS = Severity of Violence Against Women Scales, SES-SFV = Sexual Experience Survey - Short Form Victimization. *note in the full questionnaire the tactic clauses (clauses a e) are repeated with each sexual behavior item (see Table 3); other sexual behavior items were omitted for space here 
Table 2.

Sexual Experiences Scale-Romantic Partner/Non Partner (SES-RP/NP) example item

Someone had oral sex with me or made me have oral sex with them without my consent by:

How many times in an How many times not in intimate relationship? an intimate relationship?

$0, \quad 1, \quad 2, \quad 3+$ $0, \quad 1, \quad 2, \quad 3+$

Telling lies, threatening to end the relationship, threatening to spread rumors about me, making promises I knew were untrue, or continually verbally pressuring me after I said I didn't want to

Taking advantage of me when I was too drunk or out of it to stop what was happening.

Note. Only tactics a and c shown. 
Table 3.

Frequency of IPSV Endorsed

\begin{tabular}{|c|c|c|c|}
\hline & $\begin{array}{c}\text { SVAWS } \\
\text { (Samples } 1 \& 2 ; \\
n=444)\end{array}$ & $\begin{array}{l}\text { SES-SFV } \\
\text { (Sample 2; } \\
n=106)\end{array}$ & $\begin{array}{c}\text { SES-RP/NP } \\
\text { (Samples } 1 \& 2 ; \\
n=338)\end{array}$ \\
\hline $\begin{array}{l}\text { Your partner demanded sex whether you } \\
\text { wanted it or not. }\end{array}$ & $9.2 \%(41)$ & -- & -- \\
\hline $\begin{array}{l}\text { Your partner made you have oral sex } \\
\text { against your will. }\end{array}$ & $5.4 \%(24)$ & -- & -- \\
\hline $\begin{array}{l}\text { Your partner made you have sexual } \\
\text { intercourse against your will. }\end{array}$ & $5.4 \%(24)$ & -- & -- \\
\hline $\begin{array}{l}\text { Your partner physically forced you to } \\
\text { have sex. }\end{array}$ & $3.6 \%(16)$ & -- & -- \\
\hline $\begin{array}{l}\text { Your partner made you have anal sex } \\
\text { against your will. }\end{array}$ & $1.8 \%(8)$ & -- & -- \\
\hline $\begin{array}{l}\text { Your partner used an object on you in a } \\
\text { sexual way against your will. }\end{array}$ & $1.1 \%(5)$ & -- & -- \\
\hline $\begin{array}{l}\text { Someone fondled, kissed, or rubbed up } \\
\text { against the private areas of my body } \\
\text { (lips, breast/chest, crotch or butt) or } \\
\text { removed some of my clothes without my } \\
\text { consent (but did not attempt sexual } \\
\text { penetration). }\end{array}$ & -- & $9.4 \%(10)$ & $18.3 \%(62)$ \\
\hline $\begin{array}{l}\text { Someone had oral sex with me or made } \\
\text { me have oral sex with them without my } \\
\text { consent. }\end{array}$ & -- & $9.4 \%(10)$ & $7.1 \%(24)$ \\
\hline $\begin{array}{l}\text { A man put his penis into my vagina, or } \\
\text { someone inserted fingers or objects } \\
\text { without my consent. }\end{array}$ & -- & $3.8 \%(4)$ & $9.2 \%(31)$ \\
\hline $\begin{array}{l}\text { A man put his penis into my butt, or } \\
\text { someone inserted fingers or objects } \\
\text { without my consent. }\end{array}$ & -- & $2.8 \%(3)$ & $1.8 \%(6)$ \\
\hline Even though it didn't happen, someone & -- & $6.6 \%(7)$ & $11.5 \%(39)$ \\
\hline
\end{tabular}


tried to have oral sex with me, or made me have oral sex with them without my consent.

Even though it didn't happen, a man tried to put his penis into my vagina, or someone tried to stick in fingers or objects without my consent.

Even though it didn't happen, a man tried to put his penis into my butt, or someone tried to stick in objects or fingers without my consent.

$$
-5.7 \%(6) \quad 9.8 \%(33)
$$

$--\quad 1.9 \%(2)$ $4.1 \%(14)$

Note. Values reported as \%(n). IPSV = Intimate partner sexual violence; SVAWS = Severity of Violence Against Women Scales, SES-SFV = Sexual Experience Survey - Short Form Victimization; SES-RP/NP = Sexual Experiences Survey-Romantic Partner/Non-Partner 